\title{
Enhanced Sensitivities for the Searches of Neutrino Magnetic Moments through Atomic Ionization
}

\author{
Henry T. Wong, ${ }^{*}$ Hau-Bin Li, and Shin-Ted Lin \\ Institute of Physics, Academia Sinica, Taipei 11529, Taiwan \\ (Received 11 January 2010; published 2 August 2010)
}

\begin{abstract}
A new detection channel on atomic ionization for possible neutrino electromagnetic interactions is identified and studied. Significant sensitivity enhancement is demonstrated when the energy transfer to the target is of the atomic-transition scale. The interaction cross section induced by neutrino magnetic moments $\left(\mu_{\nu}\right)$ is evaluated with the equivalent photon method. A new limit of $\mu_{\nu}\left(\bar{\nu}_{e}\right)<1.3 \times 10^{-11} \mu_{B}$ at $90 \%$ confidence level is derived by using current reactor neutrino data. Potential reaches for future experiments are explored. Experiments with sub-keV sensitivities can probe $\mu_{\nu}$ to $10^{-13} \mu_{B}$. Positive observations of $\mu_{\nu}$ in this range would imply that neutrinos are Majorana particles.
\end{abstract}

DOI: 10.1103/PhysRevLett.105.061801

The compelling evidence of neutrino oscillations implies finite neutrino masses and mixings [1]. The origins and implications remain to be fully explored and understood. Experimental studies on neutrino properties and interactions can shed light on these fundamental questions and provide constraints to models on new physics.

The possible couplings of neutrinos with photons are important intrinsic properties and generic consequences of finite neutrino masses. The neutrino electromagnetic vertex can be formulated as [2-4]

$$
\Gamma^{\mu}=\frac{1}{6} q^{2}\left\langle r_{\nu}^{2}\right\rangle \gamma^{\mu}+\frac{\mu_{\nu}}{2 m_{e}} \sigma^{\mu \nu} q_{\nu}
$$

where $m_{e}$ is the electron mass and $q=(\nu ; \vec{q})$ is the fourmomentum of the virtual photon $\gamma^{*}$. The parameters $\left\langle r_{\nu}^{2}\right\rangle$ and $\mu_{\nu}$ (in units of the Bohr magneton $\mu_{B}$ ) are the "neutrino charge radius" and "neutrino magnetic moment," respectively, and describe electromagnetic couplings of neutrinos with the same and different helicities. Since different final states are involved, the two mechanisms do not interfere. Their cross sections can therefore be evaluated and studied independently. We focus on $\mu_{\nu}\left(\bar{\nu}_{e}\right)$ in this Letter and place limits with published reactor neutrino data by using an interaction channel which significantly enhances the sensitivities. There are controversial interpretations on whether $\left\langle r_{\nu}^{2}\right\rangle$ is a physical observable [5]. The contributions of $\left\langle r_{\nu}^{2}\right\rangle$ will be the subject of subsequent investigations.

The term $\mu_{\nu}$ is an effective parameter depending on the eigenstate compositions at the detectors [3]. The study of $\mu_{\nu}$ is, in principle, a way to distinguish between Dirac and Majorana neutrinos [2] - a crucial unresolved issue in neutrino physics. Early interest in $\mu_{\nu}$ was inspired by its potential relevance to explain anomalous solar neutrino data [6]. The most sensitive laboratory measurements on $\mu_{\nu}$ [4] are from neutrino-electron scattering experiments with reactor $[7,8]$ and solar neutrinos [9], where the published limits are $\mu_{\nu}\left(\bar{\nu}_{e}\right)<7.4 \times 10^{-11} \mu_{B}$ [7] and $\mu_{\nu}\left(\nu_{\odot}\right)<5.4 \times 10^{-11} \mu_{B}[9]$, at $90 \%$ confidence level
PACS numbers: 14.60.Lm, 13.15.+g, 13.40.Em, 32.80.-t

(C.L.), respectively. For exactness, the "electron" in this context should be specified to be a free electron (FE). The differential cross section is given by [3]

$$
\left(\frac{d \sigma}{d T}\right)_{\mu_{\nu}}^{\mathrm{FE}}=\frac{\pi \alpha_{e m}^{2} \mu_{\nu}^{2}}{m_{e}^{2}}\left[\frac{1-T / E_{\nu}}{T}\right]
$$

where $\alpha_{\mathrm{em}}=e^{2} / 4 \pi$ is the fine-structure constant, $m_{e}$ the electron mass, $E_{\nu}$ the neutrino energy, and $T$ the experimentally observable electron recoil energy. This expression is valid when $T$ is much larger than the atomic scale (typically $<10 \mathrm{keV}$ ). Otherwise, the target is no longer FE and the atomic binding energy $\left(\Delta_{b}\right)$ will lead to suppression in $(d \sigma / d T)^{\mathrm{FE}}$ and in the analogous standard model (SM) $\nu$-e cross section [10].

When the energy transfer is comparable to the atomic scale, a new channel corresponding to atomic ionization (AI) becomes available:

$$
\nu+(A, Z) \rightarrow \nu+(A, Z)^{+}+e^{-}(A, Z)^{+} \rightarrow(A, Z)+\gamma^{\prime} s .
$$

This is a $t$-channel process, depicted schematically in Fig. 1. The target atom $(A, Z)$ is probed and ionized electromagnetically, resembling the photoelectric effect induced

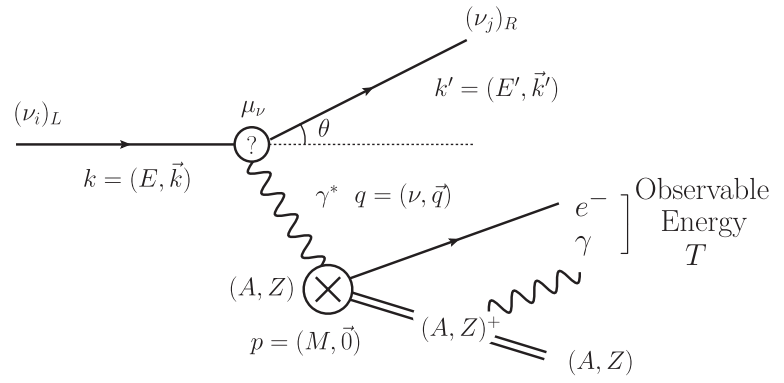

FIG. 1. Schematics of the neutrino magnetic moment interaction via the atomic ionization channel. The kinematical variables for the incoming and outgoing particles are defined in the target rest frame, following standard notations. 
by $\gamma^{*}$ instead of physical photons. Experimental observables are the kinetic energy of the ejected electron and the absorption of the subsequent $\mathrm{x}$ rays, the sum of which is $T=E_{\nu}-E_{\nu}^{\prime}$. As $T \sim \Delta_{b}$, the $q^{2} \rightarrow 0$ parameter space becomes kinematically allowed [11], as compared to $q^{2}=$ $-2 m_{e} T$ for the FE. The AI process can be described by the photoelectric cross section of real photons and is therefore enhanced. Atomic enhancement in absorption cross sections has been studied for "weakly interacting absorbable particles" like axions [12]. Resonant orbital electron capture induced by $\bar{\nu}_{e}$ has been considered for the detection of low-energy geoneutrinos [13].

The $\mu_{\nu}$-induced AI processes are analogous to the SM electromagnetic interactions (QED) between relativistic charged particles and matter which were well-studied experimentally and theoretically $[11,14]$. The QED-induced AI process dominates at atomic-scale energy as $T \sim \Delta_{b}$, as characterized by the distinct peak structures in Fig. 6 of Ref. [11]. This is the basis of relativistic charged probes being "minimum ionizing." The AI enhancement will not occur in SM $t$-channel $\nu$ - $e$ scatterings [10] or in astrophysics media with temperature high enough to be in plasma states.

The standard formalism on inelastic lepton-hadron scattering in QED [11,15,16] was adopted to derive the $\mu_{\nu}$-induced AI cross section. The kinematical variables of the neutrino probe and atomic target are defined in the target rest frame by using the standard notations shown in Fig. 1. The energy component of $\gamma^{*}$ is $\nu \equiv E_{\nu}-E_{\nu}^{\prime}=T$. The cross section can be expressed as

$$
\frac{d \sigma}{d E_{\nu}^{\prime} d \Omega}=\frac{E_{\nu}^{\prime}}{E_{\nu}} \frac{\alpha_{\mathrm{em}}^{2}}{\left(q^{2}\right)^{2}} L^{\mu \nu} W_{\mu \nu},
$$

where $L^{\mu \nu}$ is the neutrino current tensor

$$
L^{\mu \nu}=\sum_{f=L, R}\left[\bar{u}_{L}(k) \Gamma^{\mu} u_{f}\left(k^{\prime}\right)\right]\left[\bar{u}_{f}\left(k^{\prime}\right) \Gamma^{\nu} u_{L}(k)\right] .
$$

The $\mu_{\nu}$ component couples different helicity states, such that only $f=R$ contributes, giving

$$
L^{\mu \nu}\left(\mu_{\nu}\right)=\frac{\mu_{\nu}^{2}}{4 m_{e}^{2}} q^{2}\left[q^{\mu} q^{\nu}+2\left(k^{\prime \mu} k^{\nu}+k^{\prime \nu} k^{\mu}\right)\right] .
$$

The target current tensor for the AI channel, denoted by $W_{\mu \nu}$, can be parameterized with two structure functions $F_{a}\left(q^{2}, \nu\right)$ and $F_{b}\left(q^{2}, \nu\right)$ :

$$
\begin{aligned}
W_{\mu \nu}^{\mathrm{AI}}= & F_{a}\left[-\frac{q^{2}}{p \cdot q} p_{\mu} p_{\nu}-(p \cdot q) g_{\mu \nu}+p_{\mu} q_{\nu}+p_{\nu} q_{\mu}\right] \\
& +F_{b}\left[q^{2} g_{\mu \nu}-q_{\mu} q_{\nu}\right] .
\end{aligned}
$$

Accordingly, Eq. (4) becomes

$$
\begin{aligned}
\left(\frac{d \sigma}{d E_{\nu}^{\prime} d \Omega}\right)^{\mathrm{AI}}= & \frac{E_{\nu}^{\prime}}{E_{\nu}} \frac{\alpha_{\mathrm{em}}^{2}}{\left(q^{2}\right)^{2}} \frac{\mu_{\nu}^{2}}{4 m_{e}^{2}} 4\left(q^{2}\right)^{2} \frac{(p \cdot k)^{2}}{(p \cdot q)} \\
& \times\left[F_{a}\left(-1+\frac{p \cdot q}{p \cdot k}\right)-F_{b} \frac{1}{4} \frac{p \cdot q}{(p \cdot k)^{2}} q^{2}\right] .
\end{aligned}
$$

We adopted the equivalent photon approximation (EPA) [17] to describe the target tensor in terms of the photoelectric cross section of physical photons $\left(\sigma_{\gamma A}\right)$ [18]. This is justified since $q^{2} \rightarrow 0$ at $T \sim \Delta_{b}$, such that $\gamma^{*}$ is almost real. This approach has been used in studies on other $\mu_{\nu}$-induced inelastic processes [16,19]. As $q^{2} \rightarrow 0$, the total virtual photon cross section $\left(\sum_{\lambda} \sigma_{\gamma_{\lambda}^{*}}\right)$ is dominated by contributions from the two transverse components $(\lambda=$ $\pm)$, such that $[15,16]$

$$
\begin{aligned}
2 \sigma_{\gamma A} & =\left(\sigma_{\gamma_{+}^{*}}+\sigma_{\gamma_{-}^{*}}\right) \rightarrow \sum_{\lambda} \sigma_{\gamma_{\lambda}^{*}} \\
& =\frac{4 \pi^{2} \alpha_{\mathrm{em}}}{\nu}\left(-g^{\mu \nu} W_{\mu \nu}^{\mathrm{AI}}\right), \\
\text { giving } \sigma_{\gamma A} & \simeq \frac{1}{2}\left[\frac{4 \pi^{2} \alpha_{\mathrm{em}}}{\nu} 2(p \cdot q) F_{a}\right] .
\end{aligned}
$$

By combining Eqs. (8) and (9), substituting $(p \cdot q)=$ $M \nu$ and $(p \cdot k)=M E_{\nu}$ in the laboratory frame where $M$ is the target mass, and taking the leading term using $(\nu=$ $T) \ll E_{\nu}$ and $F_{b} q^{2} \rightarrow 0$, the $\mu_{\nu}$-induced AI cross section is

$$
\left(\frac{d \sigma}{d T}\right)_{\mu_{\nu}}^{\mathrm{AI}} \simeq \mu_{\nu}^{2} \frac{\alpha_{\mathrm{em}}}{\pi}\left(\frac{E_{\nu}}{m_{e}}\right)^{2} \frac{1}{T} \sigma_{\gamma A}\left(E_{\gamma}=T\right)
$$

The $\sigma_{\gamma A}(T) / T$ dependence of the cross section implies that large sensitivity enhancement with detectors at the sub$\mathrm{keV}$ threshold can be expected.

The cross sections on the Ge target due to the AI and FE channels with reactor neutrinos at $\mu_{\nu}=10^{-10} \mu_{B}$ are displayed in Fig. 2, together with the SM $\bar{\nu}_{e}-e$ [20] and $\bar{\nu}_{e}-N$ coherent [21] scatterings. The realistic spectrum [7] at a typical flux of $\phi\left(\bar{\nu}_{e}\right)=10^{13} \mathrm{~cm}^{-2} \mathrm{~s}^{-1}$ was used. The peak structures for $\mathrm{AI}$ are consequences of the enhancement of $\sigma_{\gamma A}$ [18]. The AI event rates exceed those of FE by several orders of magnitude at atomic-scale energy, reproducing

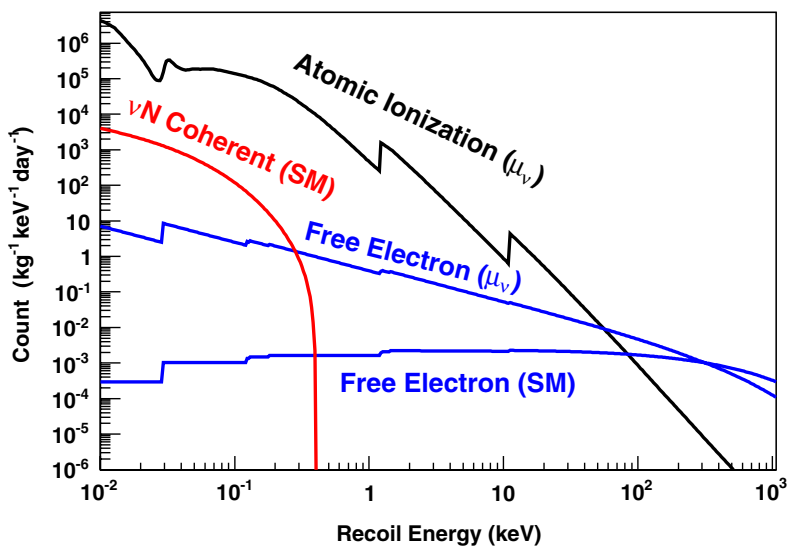

FIG. 2 (color online). The observable spectra due to neutrino interactions on the Ge target with reactor $\bar{\nu}_{e}$ at $\phi\left(\bar{\nu}_{e}\right)=$ $10^{13} \mathrm{~cm}^{-2} \mathrm{~s}^{-1}$. Contributions from the AI and FE channels at $\mu_{\nu}=10^{-10} \mu_{B}$, as well as from SM $\bar{\nu}_{e}-e$ and $\bar{\nu}_{e^{-N}}$ coherent scattering, are shown. 
similar behavior as in the QED case with charged probes [11]. The lepton energy dependences, however, are different: $E_{\nu}^{2}$ versus $\sim \log \left[E_{l}\right][11,15]$ for $\mu_{\nu}$ - and QED-induced AI, respectively.

Limits on $\mu_{\nu}\left(\bar{\nu}_{e}\right)$ were derived with the AI channel by using published data taken at the Kuo-Sheng Reactor Neutrino Laboratory where $\phi\left(\bar{\nu}_{e}\right) \sim 6 \times 10^{12} \mathrm{~cm}^{-2} \mathrm{~s}^{-1}$. The published statistical and systematic errors were adopted. An additional systematic effect was due to the uncertainties in the EPA calculation for $(d \sigma / d T)^{\mathrm{AI}}$. A conservative estimate of $20 \%$ was adopted for the analysis. This follows from the results that the EPA calculation [16] on $\mu_{\nu}$-induced photodisintegration of deuterium was consistent to within $10 \%$ and $1 \%$ with alternative derivations based on the effective-range approximation and nuclear physics approach [22], respectively.

Two independent data sets were used, each of which was analyzed with a different algorithm. (I) Figure 3(a) shows the reactor on-off residual spectrum from 570.7/127.8 days of on/off data at $12 \mathrm{keV}$ analysis threshold with the 1-kg high-purity germanium (HPGe) detector [7]. The best-fit estimate on $\mu_{\nu}\left(\bar{\nu}_{e}\right)$ to the AI cross section of Eq. (10) is $\left[\mu_{\nu} /\left(10^{-11} \mu_{B}\right)\right]^{2}=-1.0 \pm 2.7$ (stat) \pm
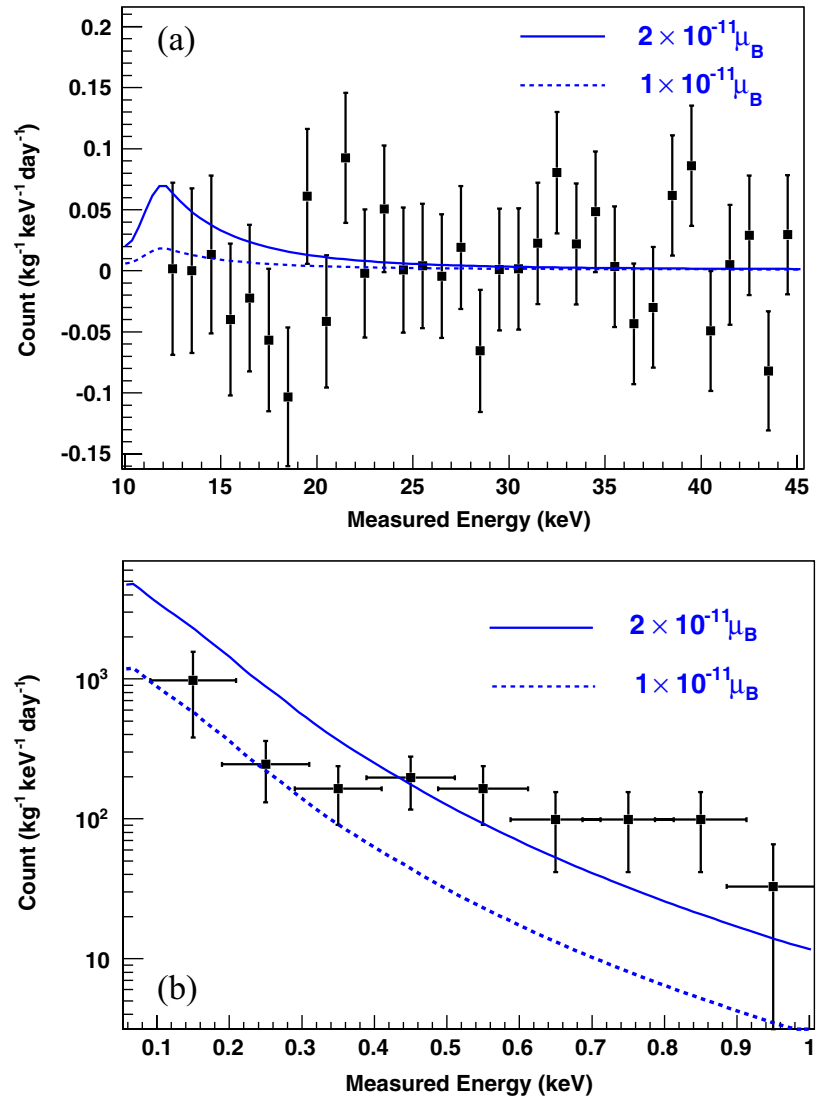

FIG. 3 (color online). (a) The reactor on-off residual spectrum from 1-kg HPGe at Kuo-Sheng Reactor Neutrino Laboratory [7]. (b) The reactor on spectrum from 20-g ULEGe [23]. The $\mu_{\nu}$-induced AI spectra at an allowed and an excluded value of $\mu_{\nu}\left(\bar{\nu}_{e}\right)$ are superimposed.
0.9 (syst) at $\chi^{2} /$ d.o.f. $=39 / 47$. This corresponds to an upper limit of

$$
\mu_{\nu}\left(\bar{\nu}_{e}\right)<1.9 \times 10^{-11} \mu_{B}
$$

at $90 \%$ C.L. (II) Figure 3(b) depicts the reactor on spectrum with $0.338 \mathrm{~kg}$-day exposure using a 20 -g ultralowenergy Ge (ULEGe) detector array at a threshold of (220 \pm 10) $\mathrm{eV}$ [23]. There was no corresponding reactor off data so that the conventional reactor on-off background subtraction was not possible. Instead, the "binned Poisson" method developed for dark matter searches, as described in Sec. 3.2 of Ref. [24], was adopted. No background assumption was made such that the $\mu_{\nu}$-induced AI contributions could not be larger than the observed signals. A more stringent limit of

$$
\mu_{\nu}\left(\bar{\nu}_{e}\right)<1.3 \times 10^{-11} \mu_{B}
$$

at $90 \%$ C.L. was derived. Both results improve over existing limits from the FE channel [7-9]. The expected $\mu_{\nu}$-induced AI spectra due to an allowed and an excluded value of $\mu_{\nu}\left(\bar{\nu}_{e}\right)\left(1 \times 10^{-11} \mu_{B}\right.$ and $2 \times 10^{-11} \mu_{B}$, respectively) are superimposed in Figs. 3(a) and 3(b) for comparison.

It is instructive to investigate the potential reach of this technique. Sensitivities are limited by ambient $\gamma /$ neutron background (AmBkg) as well as by the SM $\bar{\nu}_{e}-e$ and $\bar{\nu}_{e}-N$ processes. The projected sensitivities on $\mu_{\nu}\left(\bar{\nu}_{e}\right)$ at $\phi\left(\bar{\nu}_{e}\right)=10^{13} \mathrm{~cm}^{-2} \mathrm{~s}^{-1}$ under various background conditions are summarized in Table I. The generic goals for current projects towards observation of $\bar{\nu}_{e}-N$ coherent scattering at reactors are background levels of $\sim 1 \mathrm{~kg}^{-1} \mathrm{keV}^{-1} \mathrm{day}^{-1}$ (cpkkd) and a detection threshold of $\sim 100 \mathrm{eV}$ [21]. Assuming, in addition, that the reactor on-off subtraction of the ambient background is accurate to $\sim 1 \%, \mu_{\nu}$ can be probed to $\gtrsim 10^{-13} \mu_{B}$. Such sensitivities exceed the astrophysics bounds [4,25], typically at $\mu_{\nu}<10^{-10}-10^{-12} \mu_{B}$. In ideal experiments with AmBkg $<0.1$ cpkkd and capabilities of differentiating nuclear and electron recoil signatures, the sensitivities are limited by SM $\bar{\nu}_{e}-e$. The potential reaches are $\geq 10^{-14} \mu_{B}$ and $\geqslant 10^{-15} \mu_{B}$ at 100 and $10 \mathrm{eV}$ threshold, respectively. A similar boost in sensitivities can be expected for $\mu_{\nu}\left(\nu_{\mu}\right)$ from accelerator experiments [5], where the additional factor of $E_{\nu}^{2}$ would compensate the reduction in neutrino flux. The AI enhancement may be relevant in the search of dark matter by resonant absorption [12] or via $t$-channel scattering induced by their finite electromagnetic couplings due to magnetic moment [26] or charge [27].

The minimally extended SM with massive Dirac neutrinos predicts $\mu_{\nu} \sim 10^{-19}\left[m_{\nu} / 1 \mathrm{eV}\right] \mu_{B}$ [28], which does not produce observable consequences. Incorporation of physics such as Majorana neutrino transition moments or right-handed weak currents can significantly enhance $\mu_{\nu}$ to the experimentally relevant ranges [29]. The naturalness argument [30] dictates that Dirac neutrinos should have $\mu_{\nu} \lesssim 10^{-14} \mu_{B}$. Positive results on $\mu_{\nu}$-induced AI with 
TABLE I. Limits on $\mu_{\nu}\left(\bar{\nu}_{e}\right)$ from the FE and AI channels by using reactor neutrino data. The projected sensitivities assume $\phi\left(\bar{\nu}_{e}\right)=$ $10^{13} \mathrm{~cm}^{-2} \mathrm{~s}^{-1}$ and $<1 \%$ uncertainty in the on-off background subtraction.

\begin{tabular}{|c|c|c|c|c|}
\hline AmBkg (cpkkd) & Threshold $(\mathrm{eV})$ & Limiting background & $\mu_{\nu}\left(\bar{\nu}_{e}\right)$ sensitivities $\left(\times 10^{-12} \mu_{B}\right)$ & \\
\hline & & Mode & $\mathrm{FE}$ & AI \\
\hline \multicolumn{5}{|l|}{ Current data: } \\
\hline HPGe (Ref. [7]) & & $\mathrm{AmBkg}$ & $<57$ & $<19$ \\
\hline ULEGe (Ref. [23]) & & AmBkg & $\ldots$ & $<13$ \\
\hline \multicolumn{5}{|l|}{ Projected sensitivities: } \\
\hline 10 & 100 & $\mathrm{AmBkg}+\mathrm{SM} \bar{\nu}_{e}-N$ & 30 & 0.3 \\
\hline 1 & 100 & $\mathrm{AmBkg}+\mathrm{SM} \bar{\nu}_{e}-N$ & 10 & 0.1 \\
\hline \multirow[t]{2}{*}{$<0.1$} & 100 & $\mathrm{SM}\left(\bar{\nu}_{e^{-e}}+\bar{\nu}_{e^{-N}}\right)$ & 4 & 0.04 \\
\hline & & $\mathrm{SM} \bar{\nu}_{e}-e^{\mathrm{a}}$ & 2 & 0.009 \\
\hline \multirow[t]{2}{*}{$<0.1$} & 10 & $\mathrm{SM}\left(\bar{\nu}_{e^{-}} e+\bar{\nu}_{e^{-N}}\right)$ & 4 & 0.04 \\
\hline & & $\mathrm{SM} \bar{\nu}_{e}-e^{\mathrm{a}}$ & 0.7 & 0.0009 \\
\hline
\end{tabular}

${ }^{a}$ Denotes ideal experiments which can differentiate nuclear from electron recoils, so that the SM $\bar{\nu}_{e}-N$ background can be eliminated.

future experiments will therefore imply that neutrinos are Majorana particles. The "smoking gun" experimental signatures are observations of peak structures at the $K / L / M$ edges in the reactor on-off residual spectra, with intensity ratio $\propto\left[\sigma_{\gamma A} / T\right]$ as depicted in Fig. 2. Most background processes do not generate structures in atomic transitions. Nuclear decays by electron capture do produce peaks at the $K / L / M$ energies but at different ratios, dominated instead by captures of the $K$ orbitals.

The authors are grateful to J. W. Chen, H. N. Li, and T. C. Yuan for consultations and, in particular, to E. Akhmedov for in-depth discussions. This work is supported by the Academia Sinica Theme Project 2008-10 and through Contract No. 98-9628-M-001-013 from the National Science Council, Taiwan.

*Corresponding author. htwong@phys.sinica.edu.tw

[1] B. Kayser, Phys. Lett. B 667, 163 (2008), and references therein.

[2] B. Kayser, Phys. Rev. D 26, 1662 (1982); J. F. Nieves, Phys. Rev. D 26, 3152 (1982); R. Shrock, Nucl. Phys. B206, 359 (1982).

[3] P. Vogel and J. Engel, Phys. Rev. D 39, 3378 (1989); J. F. Beacom and P. Vogel, Phys. Rev. Lett. 83, 5222 (1999).

[4] H. T. Wong and H. B. Li, Mod. Phys. Lett. A 20, 1103 (2005), and references therein.

[5] P. Vogel and A. Piepke, Phys. Lett. B 667, 521 (2008), and references therein.

[6] A. Cisneros, Astrophys. Space Sci. 10, 87 (1971); M. B. Voloshin, M. I. Vysotsky, and L. B. Okun, Sov. Phys. JETP 64, 446 (1986).

[7] H. B. Li et al., Phys. Rev. Lett. 90, 131802 (2003); H. T. Wong et al., Phys. Rev. D 75, 012001 (2007).

[8] Z. Daraktchieva et al., Phys. Lett. B 615, 153 (2005); A. G. Beda et al., arXiv:0906.1926.

[9] C. Arpesella et al., Phys. Rev. Lett. 101, 091302 (2008).

[10] S. A. Fayans, V. Yu. Dobretsov, and A. B. Dobrotsvetov, Phys. Lett. B 291, 1 (1992); S. A. Fayans, L. A.
Mikaelyan, and V. V. Sinev, Phys. At. Nucl. 64, 1475 (2001); G. J. Gounaris, E. A. Paschos, and P.I. Porfyriads, Phys. Lett. B 525, 63 (2002).

[11] W. W. M. Allison and J. H. Cobb, Annu. Rev. Nucl. Part. Sci. 30, 253 (1980), in particular, Eq. 28 and Figs. 5 and 6.

[12] S. Dimopoulos, G. D. Starkman, and B. W. Lynn, Mod. Phys. Lett. A 1, 491 (1986).

[13] L. M. Krauss, S. L. Glashow, and D. N. Schramm, Nature (London) 310, 191 (1984).

[14] H. Bichsel, D. E. Groom, and S. E. Klein, Phys. Lett. B 667, 267 (2008), and references therein.

[15] F. Halzen and A. D. Martin, Quarks and Leptons (Wiley, New York, 1984), Chaps. 8.3-8.5; W. Greiner and J. Reinhardt, Quantum Electrodynamics (Springer-Verlag, Berlin, 1994), Example 3.17.

[16] J. A. Grifols, E. Masso, and S. Mohanty, Phys. Lett. B 587, 184 (2004).

[17] C. F. Weizsäcker, Z. Phys. 88, 612 (1934); E. L. Williams, Phys. Rev. 45, 729 (1934).

[18] B. L. Henke, E. M. Gullikson, and J. C. Davis, At. Data Nucl. Data Tables 54, 181 (1993).

[19] L. M. Sehgal and A. Weber, Phys. Rev. D 46, 2252 (1992).

[20] M. Deniz et al., Phys. Rev. D 81, 072001 (2010).

[21] H. T. Wong et al., J. Phys. Conf. Ser. 39, 266 (2006); P. A. Barbeau, J. I. Collar, and O. Tench, J. Cosmol. Astropart. Phys. 09 (2007) 009.

[22] E. Kh. Akhmedov and V. V. Berezin, Z. Phys. C 54, 661 (1992); K. Tsuji et al., Phys. Lett. B 602, 60 (2004).

[23] S. T. Lin et al., Phys. Rev. D 79, 061101(R) (2009).

[24] C. Savage et al., J. Cosmol. Astropart. Phys. 04 (2009) 010 .

[25] G. G. Raffelt, Astrophys. J. 365, 559 (1990); A. Heger et al., Astrophys. J. 696, 608 (2009).

[26] K. Sigurdson et al., Phys. Rev. D 70, 083501 (2004).

[27] S. Dimopoulos et al., Phys. Rev. D 41, 2388 (1990).

[28] B. W. Lee and R. E. Shrock, Phys. Rev. D 16, 1444 (1977).

[29] M. B. Voloshin, Sov. J. Nucl. Phys. 48, 512 (1988); R. Barbieri and R. N. Mohapatra, Phys. Lett. B 218, 225 (1989); K. S. Babu and R. N. Mohapatra, Phys. Rev. D 42, 3778 (1990).

[30] S. Davidson, M. Gorbahn, and A. Santamaria, Phys. Lett. B 626, 151 (2005); N.F. Bell et al., Phys. Rev. Lett. 95, 151802 (2005); Phys. Lett. B 642, 377 (2006). 